\title{
Preface to Special Issue on Wireless Body Area Networks: Based on Bodynets 2018 Conference
}

\author{
Matti Hämäläinen ${ }^{1} \cdot$ Daisuke Anzai $^{2} \cdot$ Giancarlo Fortino $^{3} \cdot$ Jari linatti $^{1} \cdot$ Lorenzo Mucchi $^{4} \cdot$ Carlos Pomalaza-Raez $^{5}$
}

Published online: 4 February 2020

(c) Springer Science+Business Media, LLC, part of Springer Nature 2020

In October 2018, the 13th EAI International Conference on Body Area Networks, BODYNETS 2018, was organized in Oulu, Finland. The conference was a venue for researchers from all around the world to present their latest work on various areas of wireless body area networks (WBAN). Based on the review evaluations, small amount of selected authors were invited to submit extended versions of the papers they presented in the conference to this Special Issue.

Wearable electronics, reliable wireless communications and remote care approach jointly with personal and individualized health management are the future trends modern healthcare is going to. Making this happen, new gadgets, processes and technology are needed to provide trustworthy communications mechanisms starting from data origin (user data) to electrical/medical health databases for final savings. WBANs can be seen as one important element in this whole process. Not only on-body devices, but advanced biocompatible contraptions that could seamlessly provide even in-body information were in focus of BODYNETS 2018. Dependable communications associated with accurate localization information, medical big data and other data management can greatly benefit the use of wearable technology in healthcare practices. The final goal is to make the healthcare processes more effective, reduce the medical professionals' workload and at the end, minimize the overall healthcare costs.

For this Special Issue, we have selected four articles. The topics cover new view of the future hospital; the use of ultra wideband technology in positioning in sport; on-body

Matti Hämäläinen

matti.hamalainen@oulu.fi

1 Centre for Wireless Communications, University of Oulu, Oulu, Finland

2 Nagoya Institute of Technology, Nagoya, Japan

3 University of Calabria, Calabria, Italy

4 University of Florence, Florence, Italy

5 University of Purdue, West Lafayette, USA antenna designed for communications with capsule endoscope; and finally acoustic-based security solution for WBAN communications.

The first paper on "Wireless Communications for the Hospital of the Future: Requirements, Challenges and Solutions" by Mr. Iqrar Ahmed et.al. is a conceptual presentation of the hospital of the future $(\mathrm{HoF})$. The authors are envisioning which kind of technologies the HoF could utilize. To provide secure and reliable operation environment, the use of optical communications, especially visible light communications (VLC) is highlighted. In addition, in authors' vision, there is a hybrid solution where radio and optical based wireless systems can work also in the WBAN context.

The second paper on "Ultra-wide Band Positioning in Sport: How the Relative Height Between the Transmitting and the Receiving Antenna Affects the System Performance" by Dr. Alessio Martinelli et.al. is focusing on locating football players in the field. The authors proposed a technology, which utilizes body worn ultra wideband (UWB) transmitters used by the players and static UWB receivers located in the corners of the field. The differences in location accuracies are calculated using different receiver antenna heights.

The third paper on "On-body Cavity-backed Low-UWB Antenna for Capsule Localization" by Ms. Chaïmaâ Kissi, et.al. discusses on low-band UWB on-body antenna characteristics. The antenna under discussion was designed for communications with capsule endoscope, thus directivity towards a body is a key feature. The operation frequency is between 3.75 and $4.24 \mathrm{GHz}$. The antenna utilizes cavity to get the desired directivity.

The fourth paper on "Acoustic-based Security: a key enabling technology for Wireless Sensor Networks" by Dr. Simone Soderi presents a watermark-based physical layer security protocol (WBPLSec) to be used in WBAN and other short-range applications to improve the communications security. The performance of an acoustic air-gap based WBPLSec protocol is tested in the environment, where each sensor node includes microphone and loudspeaker. It was shown that vital information can be conveyed securely through the studied channel. 


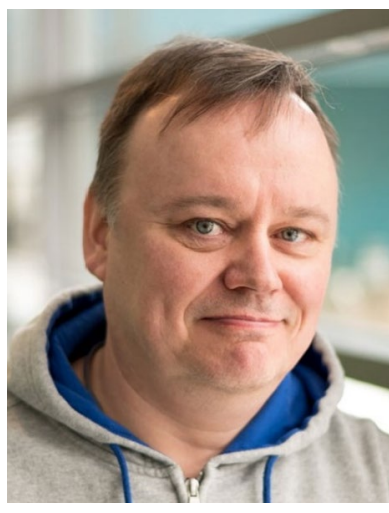

Dr. Matti Hämäläinen received his M.Sc. and Dr.Sc. degrees in 1994 and 2006, respectively, from University of Oulu, Oulu, Finland. His research interests are radio channel modelling, UWB systems, wireless body area networks and medical ICT. $\mathrm{He}$ has about 200 international scientific journal and conference publications. He is a co-author of "Wireless UWB Body Area Network s - U s ing th e IEEE802.15.4-2011", Academic Press and co-editor of "UWB: Theory and Applications", Wiley \& Sons. He holds one patent. Currently he works as a University Researcher and Adjunct Professor at Centre for Wireless Communications, University of Oulu, Finland. During 2016-2018 he was an IAS Visiting Professor at Yokohama National University, Yokohama, Japan. $\mathrm{He}$ is a member of External Advisory Board of Macquarie University's WiMed Research Centre, Australia; and International Steering Committee of International Symposium on Medical ICT (ISMICT). He was the General Chair of the 13th EAI International Conference on Body Area Networks, Bodynets 2018 hold in Oulu. He is also active contributor in ETSI TC SmartBAN. Dr. Hämäläinen is an Associate Editor of IEEE Access and member of the Editorial Board of Annals of Telecommunications. According to Google Scholar, his current H-index is 25. He is also IEEE Senior Member and EAI Community Fellow.

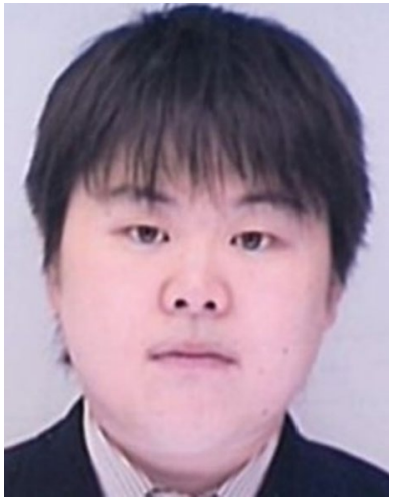

Daisuke Anzai received the B.E., M.E. and Ph.D. degrees from Osaka City University, Osaka, Japan in 2006, 2008 and 2011, respectively. Since 2011, he has been with the Graduate School of Engineering, Nagoya Institute of Technology, Nagoya, Japan, as an Assistant Professor, and where he is currently an Associate Professor. He received many awards, including the IEEE 2015 IEEE MTT-S Japan Young Engineer Award and the Telecommunications Technology Award from the Telecommunications Advancement Foundation. He has engaged in the research of biomedical communication systems and localization systems in wireless communication networks. He is also a member of European Telecommunications Standards Institute (ETSI) Technical Committee (TC) Smart Body Area Network (SmartBAN).

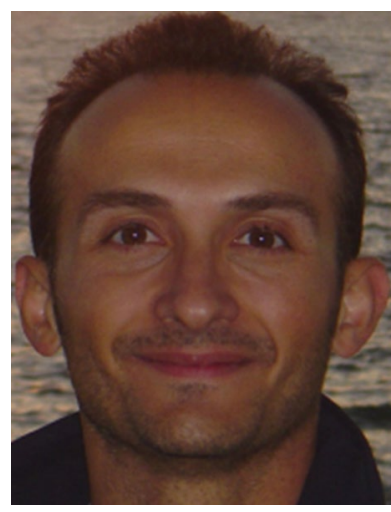

Giancarlo Fortino (SM'12) is Full Professor of Computer Engineering at the Dept of Informatics, Modeling, Electronics, and Systems of the University of Calabria (Unical), Italy. He received a Ph.D. in Computer Engineering from Unical in 2000. He is also distinguished professor at Wuhan University of Technology and Huazhong Agricultural University (China), high-end expert at HUST (China), senior research fellow at the ICAR-CNR Institute, and CAS PIFI visiting scientist at SIAT - Shenzhen. He is the director of the SPEME lab at Unical as well as co-chair of Joint labs on IoT established between Unical and WUT and SMU and HZAU Chinese universities, respectively. His research interests include agent-based computing, wireless (body) sensor networks, and IoT. He is author of $400+$ papers in int'l journals, conferences and books. He is (founding) series editor of IEEE Press Book Series on Human-Machine Systems and EiC of Springer Internet of Things series and AE of many int'l journals such as IEEE TAC, IEEE THMS, IEEE IoTJ, IEEE SJ, IEEE SMCM, Information Fusion, JNCA, EAAI, etc. He is cofounder and CEO of SenSysCal S.r.l., a Unical spinoff focused on innovative IoT systems. Fortino is currently member of the IEEE SMCS BoG and of the IEEE Press BoG, and chair of the IEEE SMCS Italian Chapter.

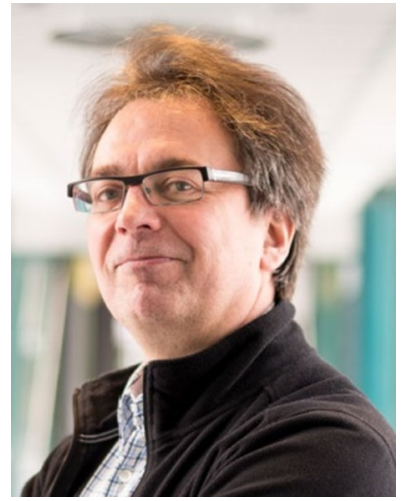

Dr. Jari linatti received M.Sc., Lic. Tech. and Dr. Tech. degrees in electrical engineering from the University of Oulu, Oulu, Finland, in 1989, 1993 and 1997, respectively. During 1989-1997 he was a Research Scientist at the Telecommunication Laboratory at the University of Oulu. During 1997-2002 he was acting professor of Digital Transmission Techniques, and senior research scientist, project manager and research director at Centre for Wireless Communications (CWC) at the University of Oulu. Since 2002 he has been Professor of Telecommunication Theory and is currently Head of CWC-Networks and Systems as well as Dean of Education at the Faculty of Information Technology and Electrical Engineering. He was IAS Visiting Professor at Yokohama National University during 2016-2018. His research interests include future wireless communication systems, transceiver algorithms, and medical ICT. Prof. Iinatti has published around 250 International Journal and Conference papers, holds six patents, and is a co-author of a book "Wireless UWB Body Area Networks: Using the IEEE802.15.4-201", Elsevier/Academic Press, a co-editor of book "UWB: Theory and Applications", Wiley \& Sons. He has been technical program committee (TPC) member in about 25 conferences, and he was TPC co-chair in IEEE PIMRC'2006, TPC chair in the ISMICT2007, general co-chair in the ISMICT2011 and ISMICT2014, and TPC Program Track CoChair in BodyNets 2012, PIMRC 2014 and PIMRC 2020, as well as TPC Co-Chair in BodyNets 2018. He was also organizer of FEELIT 2008 and FEELIT 2011 and UWBAN2012, 2013 and 2014. He serves as Steering Committee co-chair of ISMICT series. 


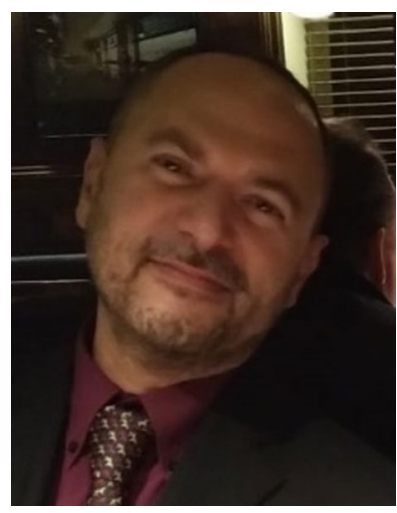

Lorenzo Mucchi (M'98-SM'12) Received the Laurea in telecommunications engineering and the Ph.D. in telecommunications and information society from the University of Florence, Italy, in 1998 and in 2001, respectively. $\mathrm{He}$ is an Associate Professor at the University of Florence, Italy. His research interests involve theory and experimentation of wireless systems and networks including physical-layer security, visible light communications, ultra wideband techniques, body area networks, and interference

management. Dr. Mucchi is serving as an associate editor of IEEE Communications Letter and IEEE Access, and he has been Editor-inChief for Elsevier Academic Press. He is a member of the European Telecommunications Standard Institute (ETSI) Smart Body Area Network (SmartBAN) group (2013) and team leader of the special task force 511 (2016) "SmartBAN Performance and Coexistence Verification." He has been lead organizer and general chair of IEEE and EAI international conferences.

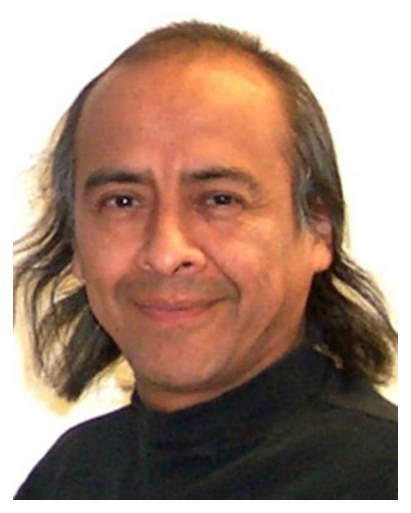

Carlos Pomalaza-Ráez is an electrical engineering professor at Purdue University, Fort Wayne, Indiana, USA. He received a B.S.M.E. and a B.S.E.E. degree from Universidad Nacional de Ingeniería Lima, Perú in 1974, and the M.S. and Ph.D. degrees in electrical engineering from Purdue University, West Lafayette, IN. He has been a faculty member of the University of Limerick, Ireland, and of Clarkson University, Potsdam, New York. He has also been a member of the technical staff at the Jet Propulsion Laboratory of the California Institute of Technology. In 2003 and 2004, under the auspices of a Nokia-Fulbright Scholar Award, he was a visiting professor at the Centre for Wireless Communications, University of Oulu, Finland. His research interests are wireless communications networks, and signal processing applications. 